\title{
Conceptos jurídicos de Derecho subjetivo \\ Legal concepts of subjective Right
}

\author{
María Beatriz Arriagada* \\ Universidad Diego Portales \\ maria.arriagada@udp.cl
}

Recibido / received: 12/09/2016

Aceptado / accepted: 20/09/2016

\section{DOI: http://dx.doi.org/10.20318/eunomia.2016.3285}

\begin{abstract}
Resumen
El objetivo de este trabajo es identificar y caracterizar brevemente los conceptos básicos o elementales de derecho subjetivo y algunas de sus posibles combinaciones, en el entendido que tales conceptos o sus combinaciones están siempre implicados en los diversos usos de la expresión "derecho subjetivo" en los discursos jurídicos. Para lograr este objetivo, el trabajo se divide en cuatro secciones. La primera sección establece que un concepto jurídico de derecho subjetivo se define por su fundamento jurídico, y que éste debe distinguirse de los fundamentos extrajurídicos que justifican o pueden justificar su otorgamiento por el ordenamiento jurídico. En la segunda sección, se analiza el modelo explicativo hohfeldiano de las posiciones jurídicas subjetivas que sirve de base para la identificación y caracterización de los conceptos jurídicos de derecho subjetivo que se realiza en la tercera sección. La cuarta sección presenta algunas consideraciones finales.
\end{abstract}

\section{Palabras clave}

Derecho subjetivo, derechos, posiciones jurídicas subjetivas, Hohfeld, normas regulativas, normas de competencia, obligaciones correlativas, potestades jurídicas.

\begin{abstract}
The aim of this paper is to identify and briefly characterize the basic or elementary concepts of subjective right and some of its possible combinations, on the understanding that such concepts or its combinations are always involved in the various uses of the term "subjective right" in legal discourse. To achieve this goal, the work is divided into four sections. The first section provides that a legal concept of rights is defined by its legal basis, and it must be distinguished from extra-legal grounds that justify or can justify the granting by law. In the second section, I analize Hohfeld's explanatory model of subjective legal positions that serves as the basis for the identification and characterization of the legal concepts of subjective right that is performed in the third section. The fourth section presents some final considerations.
\end{abstract}

\footnotetext{
* Profesora de Teoría del derecho de la Universidad Diego Portales. Santiago de Chile. Correos electrónicos: mbeatriz.arriagada@gmail.com; maria.arriagada@udp.cl. Este trabajo corresponde a resultados del proyecto Fondecyt de iniciación $N^{\circ} 11150372$ "Análisis de la tensión entre el concepto y el fundamento de los derechos fundamentales a partir de las ideas de Fernando Atria sobre las diferencias entre los derechos civiles y políticos y los derechos sociales", financiado por el Fondo Nacional de Desarrollo Científico y Tecnológico del Gobierno de Chile. El presente trabajo ha sido desarrollado en conjunto con Álvaro Núñez y Claudio Agüero. Aunque la firma es individual y tenemos algunos desacuerdos menores, es producto de un trabajo de reflexión conjunto.
} 


\section{Keywords}

Subjective right, rights, subjective legal positions, Hohfeld, regulative norms, competence norms, correlative duties, legal power.

SUMARIO. 1. Conceptos y fundamentos a propósito de los derechos subjetivos 2. Bases para la identificación y caracterización de los conceptos jurídicos de derecho subjetivo 3 . Conceptos jurídicos de derecho subjetivo 4. Algunas consideraciones finales.

1. Conceptos y fundamentos a propósito de los derechos subjetivos

La expresión "derecho subjetivo" (o simplemente "derechos") no se usa exclusivamente en el discurso de las fuentes del derecho y en los meta-discursos que a él se refieren, como la dogmática jurídica, la teoría del derecho y la metateoría del derecho. Aunque aquí sólo haré referencia al uso de esta expresión en contextos jurídicos, conviene aclarar que es perfectamente plausible hablar de derechos subjetivos morales, con la condición de que con ello se aluda a atributos, situaciones o posiciones subjetivas activas fundamentadas en normas morales.

Ello supone aceptar, en primer lugar, que los derechos no son atributos naturales sino artificiales. Esto significa que los derechos son conferidos por normas que pueden ser jurídicas o morales y, en consecuencia, que los derechos no existen antes que las normas (Guastini, 1999: 180-182). En términos jurídicos (que desde luego pueden extenderse al discurso moral), una posición o situación subjetiva es la dimensión subjetiva de una norma, es decir, lo que la norma adscribe a un sujeto, por ejemplo, un derecho, un permiso o un deber (Guastini, 2016: 83). Es la forma subjetiva de manifestarse la norma (Kelsen 1987 [1923]: 539-540).

En segundo lugar, asumo que decir que alguien tiene un derecho siempre implica hablar de o desde un determinado sistema jurídico o moral (Mackie, 2000 [1977]: 198) ${ }^{1}$. Esto implica que los derechos se fundamentan en normas (jurídicas o morales) cuya existencia es contingente y empíricamente demostrable.

Como en contextos jurídicos, la expresión derecho subjetivo se usa en variados sentidos ${ }^{2}$, el objetivo de este trabajo es identificar y caracterizar brevemente los conceptos básicos o elementales de derecho subjetivo y algunas de sus posibles combinaciones. Este esfuerzo se justifica porque tales conceptos o las combinaciones de éstos están siempre implicados en los diversos usos que, de la expresión derecho subjetivo, se hace en los discursos jurídicos.

La clave para que este esfuerzo sea exitoso es comprender que lo que define un concepto jurídico o tipo jurídico de derecho subjetivo es su fundamento jurídico. Esto es la norma o normas del derecho objetivo que atribuyen a una clase de sujetos una posición jurídica subjetiva activa y que, en este sentido, la fundamentan.

\footnotetext{
${ }^{1}$ Porque no es posible sostener fundadamente la existencia de derechos humanos naturales puesto que no puede darse por sentada la existencia de una naturaleza humana intrínseca (Arendt, 2003 [1958]: 24-25 y Rorty, 1991 [1989]: 27) y tampoco es demostrable la existencia de derechos humanos universales, objetivamente válidos y cognoscibles por los seres humanos (Bulygin, 1991 [1987]: 622623 y 625$)$.

2 Esto no es solamente verdad por el hecho de que el uso de la expresión "derecho subjetivo" ha ido mutando a lo largo de la historia sino por el hecho de que ella se usa, sincrónicamente, en muy diferentes sentidos.
} 
La tarea de identificar el fundamento jurídico de los diferentes tipos de derecho subjetivo es completamente diferente de la de identificar las razones morales extra-jurídicas que justifican su atribución por el ordenamiento jurídico. Como sugiere Kelsen (1987 [1923]: 507 y 539-540), el derecho no es sustancia, sino forma. No es lo protegido, sino la protección. Observadas las normas desde su dimensión subjetiva, el derecho (subjetivo) es la posición o situación jurídica y la norma es su fundamento.

Un derecho subjetivo jurídico puede y suele ser, al mismo tiempo, un derecho subjetivo moral. Esto es así porque los sistemas jurídicos suelen reflejar la moral mayoritaria, o al menos dominante, de la comunidad jurídica respectiva. En consecuencia, se puede afirmar que para cada concepto de derecho subjetivo hay dos clases de fundamento: (i) un fundamento jurídico y (ii) un fundamento moral. A la teoría del derecho le interesa el primero. A la filosofía política del derecho le interesa el segundo.

Así, la disputa entre las llamadas teorías de la voluntad y del interés -cuyos exponentes más conocidos son Windscheid (1946 [1862]: 219 y ss.) ${ }^{3}$ y lhering (2011 [1865]: 816 y 821$)^{4}-{ }^{5}$, y más tarde Hart (1962 [1953]: 117-119; 1982:183-188) y algunos de sus discípulos (MacCormick, 1988 [1975], Raz, 2001 [1994]: 57-68)- es un enfrentamiento de dos perspectivas diferentes acerca del fundamento extrajurídico para conferir derechos subjetivos (Arriagada, 2014: 27-39) ${ }^{6}$.

Ambas perspectivas y cualquier combinación de ellas incurren en el error de confundir la protección conferida por las normas del derecho con los bienes que son objeto de esa protección y, en consecuencia, no son realmente teorías sino ideologías. Un derecho subjetivo no es una voluntad o un interés jurídicamente protegido. Es la protección de esa voluntad o de ese interés, es decir, la posición jurídica subjetiva.

2. Bases para la identificación y caracterización de los conceptos jurídicos de derecho subjetivo

Hohfeld (1992 [1913]: 31, 47-52, 67-68 y 81) comprendió bien la importancia de distinguir el fundamento jurídico del fundamento extra-jurídico de los derechos subjetivos. Su modelo explicativo de las diferentes posiciones jurídicas subjetivas (activas y pasivas) a las que comúnmente se alude con las expresiones de "derecho subjetivo" y "deber" se funda precisamente en la relevancia de distinguir las relaciones puramente jurídicas de los hechos físicos y psíquicos que las hacen surgir.

Sobre esta base, identificó dos grandes grupos de posiciones jurídicas subjetivas que se definen por sus correlativos y opuestos, es decir, en términos relacionales:

\footnotetext{
${ }^{3}$ Párrafo 37, Libro I, Tomo 1.

${ }_{5}^{4}$ Libro II, Segunda parte, Título I.

5 La idea de derecho subjetivo como un poder o señorío de la voluntad tiene, no obstante, antecedentes anteriores a Windscheid; ver, por ejemplo, Hobbes (1980) [1651]: 106 y 118-119 (Capítulos XIV y XV); Spinoza (2003) [1670]: 334-352 (Capítulo XVI) y Kant (2005) [1797]: 41-42 (párrafo $\mathrm{N}^{\circ}$ 232). De hecho, específicamente a Kant y a su escuela se dirige la crítica de Ihering, a quien sin duda puede atribuirse el origen de la llamada teoría del interés. Sobre esta atribución, ver Villey (1976) [1968]: 229-233.

${ }^{6}$ Un buen resumen de la discusión de las llamadas teorías de la voluntad y del interés puede verse, por ejemplo, en Finnis (2000) [1980]: 231-233. Una descripción de ambas teorías y asimismo de las teorías mixtas, Dabin (2006) [1955]: 67-94.
} 
a.- Las posiciones jurídicas subjetivas del primer grupo son las siguientes: (i) la posición que propiamente debe ser denominada derecho subjetivo o pretensión se caracteriza porque su correlativo es un deber en sentido propio y su opuesto un no derecho; (ii) la situación de libertad o privilegio se caracteriza porque su correlativo es un no derecho, y su opuesto un deber en sentido propio; (iii) la posición que propiamente se denomina deber se caracteriza porque su correlativo es un derecho y su opuesto una libertad o privilegio; y (iv) la posición de no derecho se caracteriza porque su correlativo es una libertad o privilegio y su opuesto un derecho.

b.- Las posiciones del segundo grupo son las siguientes: (i) la posición de potestad o competencia se caracteriza porque su correlativo es una sujeción, y su opuesto una incompetencia; (ii) la situación de inmunidad se caracteriza porque su correlativo es una incompetencia, y su opuesto una sujeción; (iii) la posición de sujeción se caracteriza porque su correlativo es una potestad o competencia y su opuesto una inmunidad; y (iv) la situación de incompetencia se caracteriza porque su correlativo es una inmunidad y su opuesto una potestad o competencia.

La utilidad de este modelo de análisis se justifica principalmente por dos razones. La primera razón es su carácter "conectivo" (la comprensión apropiada de cada concepto se obtiene captando sus relaciones con los demás conceptos del mismo sistema) ${ }^{7}$. La segunda razón es que a este modelo subyacen (y en este sentido, el modelo anticipa) dos distinciones que han demandado mucho trabajo e inteligencia a los teóricos del derecho y que han demostrado un rendimiento explicativo difícil de exagerar. La primera distinción es entre tipos de normas: (i) normas prescriptivas o regulativas, y (ii) normas de competencia o sobre la producción jurídica. La segunda distinción es entre tipos de acciones humanas: (i) acciones puramente físicas o materiales, y (ii) acciones que, siendo físicas o materiales son, además, normativas (Hart 2009 [1961]: capítulos III y V, Ross, 2000 [1968)]: 68-72, Alchourrón y Bulygin 1991 [1983], Bulygin 1981 [1988]) ${ }^{8}$.

Sin que sea posible entrar en el debate que acompaña al esfuerzo por trazar la distinción entre tipos de normas ${ }^{9}$, trabajaré con las siguientes definiciones estipulativas:

a.- Las normas regulativas o prescriptivas son aquellas que, en un sistema jurídico, ordenan o permiten la realización o abstención de determinadas acciones o la consecución o no consecución de determinados fines ${ }^{10}$.

b.- Las normas de competencia o sobre la producción jurídica son aquellas que, en un sistema jurídico, definen las competencias o potestades para producir

\footnotetext{
7 Sobre este tipo de análisis, ver Strawson, 1997 [1992]: 63. Y sobre la caracterización de la explicación hohfeldiana como un análisis de este tipo, ver Nino, 1980 y Cruz Parcero, 2007: 33-34 (nota No19).

'Un análisis que destaca estas características del modelo hohfeldiano, aunque con matices, se encuentra en Arriagada, 2014:15-19.

${ }^{9}$ La discusión teórica es en realidad más amplia en cuanto, entre los autores que creen que las normas de competencia son de un tipo diferente e irreductible al de las normas regulativas, es usual la consideración de que las llamadas normas de competencia son una especie del género más amplio de las denominadas normas constitutivas o determinativas. Este no es el lugar para entrar en esta discusión sobre todo porque hasta ahora no existe suficiente claridad ni acuerdo sobre su significado ni sobre su campo de aplicabilidad (Searle, 2007 [1969]: 43-45 y 1997 [1995], Conte,1983, Schauer, 2004 ([1991]: 63)

${ }^{1}$ La definición coincide con la caracterización que Von Wright (1970 [1963]: 26-27 y 87-107) hace de las prescripciones o regulaciones, salvo por el hecho de que aquí se considera que el contenido de las normas no está circunscrito únicamente a acciones y actividades, puesto que la autoridad normativa puede prescribir la obtención o la abstención de la obtención de fines u objetivos.
} 
válidamente normas jurídicas o participar válidamente en su producción. En este género se incluyen las normas que atribuyen a determinados sujetos la competencia de producir normas en determinado ámbito material de regulación y las que definen las condiciones para el ejercicio de esa competencia (el procedimiento y los límites de contenido que las normas producidas no pueden sobrepasar) (Arriagada, 2015 $112-113)^{11}$.

El criterio para distinguir los tipos de acciones humanas es si su realización y las consecuencias que de su realización se siguen han sido o no definidas o constituidas por normas de competencia ${ }^{12}$ :

a.- Las acciones puramente físicas o materiales pueden ser realizadas, descritas y comprendidas sin referencia a norma alguna, aunque pueden ser reguladas por normas, en cuyo caso adquieren significado jurídico (Kelsen 1991 [1960]: 15-16) ${ }^{13}$.

b.- Las acciones son, además de físicas, normativas (producen, modifican o derogan normas) cuando su realización y las consecuencias que de su realización se siguen han sido definidas o constituidas por normas de competencia, de suerte que sólo pueden ser realizadas de acuerdo con ellas y no pueden ser descritas ni entendidas sin referencia a ellas ${ }^{14}$. Nada de esto impide que, una vez definidas, las acciones normativas sean reguladas por normas regulativas ${ }^{15}$.

Estas distinciones son fundamentales en la caracterización de los tipos de derecho subjetivo y permiten sintetizar el modelo hohfeldiano en las siguientes afirmaciones:

\footnotetext{
${ }^{11}$ Esta definición de normas de competencia asume que: (i) las expresiones "producir" y "producción" de normas incluyen la modificación y la derogación de normas; (ii) una norma es jurídicamente válida cuando ha sido producida de conformidad con las normas (de competencia) que, en un sistema jurídico, definen las potestades o competencias para producirla (Orunesu, 2012: 119-120, Guastini, 2001: 60; Kelsen 1991 [1960]: 201-208); (iii) la competencia es la posibilidad que ciertas normas de un sistema jurídico atribuyen a un sujeto (órganos legislativos, judiciales y administrativos o individuos) la posibilidad de producir o de participar en la producción de normas jurídicas válidas, en las condiciones definidas por esas mismas normas (Kelsen, 1991 [1960]: 159-161 y 151-157, Ross, 1991 [1961]: 77-78, Atienza y Ruiz Manero, 2004: 83, Hohfeld, 1992 [1913]: 71)).

12 Una distinción similar es la de "actos informales" (naturales) y "actos formales" (artificiales) propuesta Ferrajoli (2011) [2007]: 464-465. Los actos informales sólo pueden ser supuestos de hecho de normas y se caracterizan porque producen efectos de hecho que son desincentivados o incentivados por el derecho y efectos de derecho preestablecidos por las normas que los regulan. Los actos artificiales no son simplemente supuestos de hecho de normas sino también actos aplicadores de normas que producen solamente efectos de derecho condicionados por las normas que lo prevén a la observancia de ciertas formas en el ejercicio de determinados poderes.

${ }^{13}$ La acción de matar a otro, por ejemplo, puede tener el significado jurídico de "homicidio" e incluso el significado jurídico de la ejecución de una "pena de muerte", pero "matar a otro" sigue siendo una acción que puede ser realizada, descrita y comprendida sin referencia a las normas que la califican como homicidio o como pena de muerte.

14 Las acciones que el ordenamiento jurídico prevé, por ejemplo, para el otorgamiento de un testamento o la dictación de una ley o una sentencia judicial son obviamente físicas, pero sólo pueden ser realizadas de acuerdo con las normas que definen las potestades para otorgar válidamente un testamento y para dictar válidamente una ley o una sentencia.

${ }^{15}$ La facultad, potestad o capacidad de producir derecho que el orden jurídico confiere a un individuo puede ser simultáneamente obligatoria (Kelsen, 1991 [1960]: 132). Las potestades pueden ser reforzadas mediante deberes (Hart, 2009 [1961]: 37 y 120). La permisión es algo que puede darse tanto respecto de actos ordinarios como respecto de actos jurídicos (Bulygin, 1991 [1988]: 489). Una cosa es conferir un poder (normativo) y otra es regular el ejercicio de ese poder (Atienza y Ruiz Manero, 2004: 84).
} 
a.- Las posiciones jurídicas subjetivas correlativas de "derecho subjetivo" (en sentido propio) y "deber" (en sentido propio) del primer grupo se fundamentan en normas regulativas o prescriptivas.

b.- Las situaciones jurídicas subjetivas correlativas de "potestad" y "sujeción" del segundo grupo se fundamentan en normas de competencia que definen las potestades para producir, modificar o eliminar válidamente normas $\mathrm{y}$, por tanto, posiciones y relaciones normativas.

c.- La relación de "libertad-no derecho" del primer grupo y la relación de "inmunidad-incompetencia" del segundo grupo connotan, respectivamente, la ausencia de normas regulativas y la ausencia de normas de competencia, de suerte que "libertad", "no derecho", "inmunidad" e "incompetencia" no son propiamente posiciones jurídicas subjetivas adscritas a los sujetos por normas ${ }^{16}$.

d.- Mientras las relaciones de "derecho-deber" pueden referirse a la exigencia de realizar o de abstenerse de realizar acciones físicas o materiales que son supuestos de hecho de normas regulativas ${ }^{17}$, una relación de "libertad-no derecho" puede connotar la inexistencia de la exigencia de realizar este tipo de acciones pues se fundamenta en la ausencia de normas de regulativas (Arriagada, 2014: 19).

e.- Mientras las relaciones de "potestad-sujeción" se refieren siempre a la posibilidad de realizar acciones normativas en aplicación de normas de competencia, una relación de "inmunidad-incompetencia" connota la imposibilidad de realizar tales acciones porque se fundamenta en la ausencia de normas de competencia (Arriagada, 2014: 19).

\section{3.- Conceptos jurídicos de derecho subjetivo}

3.1 Los tipos básicos o elementales de derecho subjetivo

Lo hasta ahora dicho permite reconocer la existencia de dos tipos básicos o elementales de derecho subjetivo. El criterio para distinguirlos es el tipo de norma jurídica que los confiere.

\subsubsection{El derecho subjetivo en sentido propio}

El primer tipo básico de derecho subjetivo es el que Hohfeld (1992 [1913]: 47-52) llamó derecho subjetivo en sentido propio y que Kelsen (1991 [1960]: 139-143) denominó derecho reflejo. De acuerdo con este concepto, un individuo tiene un derecho subjetivo cuando, en virtud de una norma jurídica regulativa, otro u otros se encuentran a su respecto obligados a comportarse de cierta manera.

Como se trata simplemente del correlativo o el reflejo de una obligación impuesta por una norma prescriptiva, no es un derecho "activo" a hacer algo sino un

\footnotetext{
${ }^{16}$ La situación de "libertad" del primer grupo cuyo correlativo es un "no derecho" y la situación de "inmunidad" del segundo grupo cuyo correlativo es una "incompetencia" ocupan exactamente el mismo lugar en su respectivo grupo porque se trata, como sugería Hart (1962 [1953]: 118-119 y nota №14 del mismo trabajo) de "casos negativos", esto es, aquellos en que derecho reconoce negativamente la elección de un individuo porque "no hay" norma que interfiera si el individuo opta por realizar o por omitir alguna acción (libertad) o por conservar su posición jurídica inalterada (inmunidad). El propio Hohfeld (1992 [1913]: 81) manifiesta que la inmunidad es la libertad de una persona frente a la potestad jurídica o contralor jurídico de otro, con respecto a una relación jurídica.

${ }^{17}$ En tanto la posición pasiva denominada "deber" a menudo consiste en una exigencia de realizar o no una determinada conducta material.
} 
derecho pasivo a que se respete una situación o a recibir algo por parte de otro (Cruz Parcero, 2007: 24).

Esta aclaración permite, además, descartar fundadamente la idea común de que los derechos subjetivos son permisos o autorizaciones conferidos por normas para realizar o abstenerse de realizar determinadas acciones. Si los permisos en sentido fuerte (conferidos por normas permisivas) que sólo consisten en una declaración de mera tolerancia por parte de la autoridad normativa son entendidos como promesas de no interferencia, pueden considerarse prohibiciones que la autoridad se dirige a sí misma. Sin embargo, no pueden considerarse derechos, salvo que la mera tolerancia se combine con una prohibición de no interferencia dirigida a terceros, en cuyo caso el titular del permiso tiene un derecho en relación con los sujetos de la prohibición (Von Wright, 1970 [1963]: 101-107). En esta hipótesis, estos sujetos se encuentran, frente al titular del derecho, obligados a abstenerse de actuar de cierta manera, con fundamento en una norma prescriptiva.

Si la obligación correlativa es, en cambio, positiva, como, por ejemplo, las obligaciones específicas que el Estado puede tener en relación con la salud, la vivienda o la seguridad social de los individuos, éstos tienen un derecho (social) a que el Estado realice determinadas prestaciones materiales en relación con la salud, la vivienda o la seguridad social. La salud, la vivienda y la seguridad social no son derechos sino bienes que el derecho objetivo protege, por ejemplo, confiriendo ciertos derechos subjetivos que reflejan obligaciones estatales positivas (Arriagada, 2015b: 827-828).

3.1.2 El derecho subjetivo como potestad o competencia El segundo tipo básico de derecho subjetivo corresponde a la posición jurídica subjetiva activa que propiamente ha de llamarse potestad o competencia. En esta posición se encuentra el sujeto de cuya voluntad depende la creación, modificación o eliminación de normas y, en consecuencia, de posiciones y relaciones normativas (Hohfeld 1992 [1913]: 67-80). De acuerdo con este concepto, un individuo tiene un derecho subjetivo cuando, con fundamento en una norma jurídica de competencia, tiene la posibilidad de realizar actos jurídicos que producen, modifican o eliminan normas jurídicas, y, en consecuencia, posiciones y relaciones jurídicas.

El llamado "derecho al sufragio", por ejemplo, es una potestad o competencia. No es la libertad de realizar una acción que no está prohibida. Tampoco es un derecho reflejo ni un derecho subjetivo en sentido técnico porque no refleja una obligación de realizar o abstenerse de realizar determinados actos. No refleja una obligación porque no se fundamenta en normas regulativas sino únicamente en normas de competencia que, en los órdenes jurídicos democráticos, confieren a los individuos la potestad de participar en las decisiones de creación, modificación y derogación de las normas del ordenamiento jurídico (Kelsen, 1991 [1960]: 150-152, Arriagada, 2015a: 114-116) ${ }^{18}$.

\footnotetext{
${ }^{18}$ Como en los sistemas democráticos los órganos del Estado son competentes para producir normas que obligan a los sujetos sometidos al derecho precisamente porque dichos órganos son elegidos a través del sufragio de los titulares de este derecho, éste es una condición de la validez de las normas jurídicas producidas por las autoridades públicas. Una cuestión diferente es que para poder afirmar que el derecho al sufragio así entendido existe en un ordenamiento jurídico y que éste es, en consecuencia, democrático, no basta que la Constitución así lo declare. Es necesario que el Estado se abstenga de impedir su ejercicio (obligación negativa) y provea los medios jurídicos y materiales que lo hagan posible (obligaciones positivas normativas y fácticas). Admitir esto no equivale a afirmar que estas posiciones jurídicas son derechos subjetivos que reflejan obligaciones estatales de abstención y de prestación.
} 


\section{2.- Derechos subjetivos complejos}

Una situación distinta a las explicadas en el apartado anterior tiene lugar cuando al titular de un derecho subjetivo en sentido propio, se le confiere, además, la potestad o competencia de exigir judicialmente el cumplimiento de la obligación correlativa a su derecho o la ejecución de la sanción prevista para el caso de su incumplimiento ${ }^{19}$.

Kelsen (1991 [1960]: 146-149 y 152) llama a esta situación derecho subjetivo en sentido técnico. $Y$ la define como un derecho reflejo unido al poder jurídico de poner en movimiento, mediante una acción, el procedimiento destinado a reconocer el incumplimiento de la obligación por el deudor o deudores. Hart (1982: 183-184 y 188), en cambio, prefiere caracterizar esta situación como aquella en la que se encuentra un sujeto que tiene algún grado de control sobre el cumplimiento de la obligación de otro. Aunque las diferencias son de énfasis (Arriagada, 2014: 30-37) ${ }^{20}$, en ambos casos se trata de un derecho igualmente pasivo que, a diferencia del simple derecho reflejo, se encuentra judicialmente garantizado ${ }^{21}$.

Esta situación jurídica se fundamenta en dos tipos de normas diferentes: (i) una norma regulativa que impone una obligación y confiere un derecho reflejo y (ii) una norma de competencia que confiere un poder normativo. En consecuencia, la situación puede ser descrita como una conjunción de los conceptos de derecho subjetivo en sentido propio y potestad o competencia (Arriagada, 2015a: 111-112). De ahí que pueda hablarse de un tipo complejo de derecho subjetivo. El comprador, por ejemplo, no tiene sólo un derecho (reflejo) a que vendedor le entregue la cosa vendida, sino también la potestad de controlar el cumplimiento de la obligación del vendedor: tiene el poder exigirle que cumpla y el poder de liberarlo de ese cumplimiento.

El grado de control que, sobre el cumplimiento de la obligación, tiene el titular de este tipo de derecho subjetivo no es siempre el mismo. En el ámbito del derecho penal, por ejemplo, los individuos tienen un derecho subjetivo (reflejo) a no ser lesionados y también la potestad de reclamar judicialmente frente al incumplimiento de la obligación, pero no tienen el poder de liberar a los obligados (Hart, 1982: 183185). El derecho (civil) al que suele llamarse "libertad de expresión" que refleja una obligación estatal de no interferencia suele estar unido a la potestad de exigir judicialmente el cumplimiento de esa obligación, pero no incluye la opción de liberar al obligado de ese cumplimiento.

Otra posible combinación de derechos subjetivos básicos se presenta, por ejemplo, cuando los individuos con potestad para realizar determinados actos

\footnotetext{
${ }^{19}$ Poco importa si la acción está destinada a obtener el cumplimiento de la obligación incumplida o la ejecución de la sanción estatuida para el caso de incumplimiento de la obligación pues, en cualquiera de estas hipótesis, el sujeto tiene una potestad de participar en el proceso de creación judicial de una norma jurídica prescriptiva.

${ }^{20}$ La diferencia reside en que mientras Hart enfatiza el poder de control y, en consecuencia, la posibilidad de elección del titular del derecho subjetivo, Kelsen pone el acento en la existencia de una norma que confiere un poder para exigir la ejecución de la sanción en caso de incumplimiento de la obligación que el derecho refleja.

${ }^{21}$ Sobre la distinción entre los derechos y su garantía judicial, ver por ejemplo Alexy, 2007 [1984]: 456 y Guastini, 1999 [1996]: 185. Vale la pena mencionar que el concepto analizado encuentra antecedentes importantes en el lusnaturalismo racionalista. Puede verse, por ejemplo, en lo que Grocio y Pufendorf denominaron "derecho perfecto", pero sobre todo en Thomasius que, junto con precisar que el derecho y la obligación son conceptos correlativos, sostiene, en contra de la división entre derecho perfecto e imperfecto, que todo derecho es perfecto porque la obligación que a él corresponde es siempre externa y sujeta a coacción; Grocio (1987) [1625]: 53-54 (Capítulo I, III, 1 y 2 y IV); Pufendorf (2009) [1660]: 91-92 (libro I, definición VIII, 1 y 2); y Thomasius (1994) [1705]: 212-213 (capítulo V, párrafos VIII, IX, XXI-XXV).
} 
normativos tienen también derecho a no ser interferidos en el ejercicio de esa potestad. En esta hipótesis, la combinación se produce de modo inverso: el individuo tiene, con fundamento en una norma de competencia, una potestad de participar en la producción de normas (por ejemplo, el llamado derecho a voto) y, con fundamento en una norma regulativa, un derecho subjetivo a que otros no interfieran en el ejercicio de esa potestad ${ }^{22}$.

Si este derecho subjetivo de ejercitar una potestad normativa pudiere reclamarse judicialmente, estaríamos frente a una combinación de tres posiciones jurídicas activas básicas o derechos subjetivos básicos: (i) una potestad de realizar un acto normativo, con fundamento en una norma de competencia, (ii) un derecho a que nadie impida el ejercicio de esta potestad, con fundamento en una norma regulativa, y (iii) una potestad para exigir judicialmente el cumplimiento de la obligación de no interferir en el ejercicio de la potestad o la ejecución de la sanción prevista para el caso de incumplimiento, con fundamento en una norma de competencia.

\section{4.- Algunas consideraciones finales}

a.- Todas las posiciones o situaciones jurídicas subjetivas activas conferidas por normas del derecho objetivo pueden reducirse a los tipos básicos de derecho subjetivo que han sido caracterizados o a una combinación de estos tipos básicos.

b.- Las posiciones jurídicas subjetivas que han sido caracterizadas sobre la base de sus fundamentos jurídicos son compatibles con diferentes razones morales que aspiran a justificar su atribución por el ordenamiento jurídico. Cualquier discurso que afirme que la atribución de determinadas posiciones jurídicas está comprometida con determinadas doctrinas morales o políticas no es una teoría descriptiva o explicativa acerca de los derechos tal como son sino una ideología sobre lo que los derechos deben ser.

c.- La reconstrucción estándar de los llamados "derechos fundamentales" y del llamado "derecho de propiedad" en términos de un conjunto de posiciones subjetivas activas (la idea de "derechos en sentido amplio") suele conducir a la conclusión de que los derechos son bienes y, por tanto, razones que justifican normas ${ }^{23} \mathrm{y}$, en consecuencia, a la conclusión (iusnaturalista) de que los derechos existen antes que las normas.

Bibliografía

ALCHOURRÓN, C. y BULYGIN, E. (1991) [1983], "Definiciones y normas". En: ALCHOURRÓN, C., y BULYGIN, E., Análisis lógico y derecho, Madrid, CEPC, pp. 439-463.

ALEXY, R. (2007) [1984], Teoría de los derechos fundamentales (Trad. de Carlos Bernal Pulido), CEPC, Madrid.

ARENDT, H. (2003) [1958], La condición humana. (Trad. de Ramón Gil Novales), Paidos, Buenos Aires.

ARRIAGADA, M.B. (2014), "El concepto hohfeldiano de derecho subjetivo", Revista de Ciencias Sociales, núm. 65, pp. 13-45.

ARRIAGADA, M.B. (2015a), "Las cortes constitucionales frente a los 'derechos civiles y políticos'. Una mirada desde la teoría analítica del derecho", Revista

\footnotetext{
${ }^{22}$ La situación referida es diferente a la identificada por Diccioti (2004: 738) consistente en el derecho de un individuo a que otro u otros realicen un acto jurídico (normativo) de determinado contenido, del cual depende el goce o la tutela de un derecho propio, como el derecho de las partes de un juicio al pronunciamiento de una sentencia.

${ }^{23}$ Un ejemplo claro puede verse en Laporta,1987.
} 
española de derecho constitucional, núm. 105, septiembre-diciembre, pp. 105-136.

ARRIAGADA, M.B. (2015b), "La imposibilidad de equiparar derechos liberales y sociales", Revista chilena de derecho (Pontificia Universidad Católica de Chile), Volumen 42, núm. 3, pp. 819-842.

ATIENZA, M. y RUIZ MANERO, J. (2004), Las piezas del derecho, Ariel. Barcelona.

BULYGIN, E. (1991) [1987], "Sobre el estatus ontológico de los derechos humanos". En: ALCHOURRÓN, C., y BULYGIN, E., Análisis lógico y derecho, CEPC, Madrid, pp. 619-625.

BULYGIN, E. (1981) [1988], "Sobre las normas de competencia". En: ALCHOURRÓN, C. y BULYGIN, E., Análisis lógico y derecho, Madrid, CEPC, pp. 485-498.

CONTE, A. G. (1983): "Regola Costitutiva, Condizione, Antinomia". En: SCARPELLI, $\cup$ (ed.). La teoria generale del diritto. Problemi e tendente attuali. Studi dedicati a Norberto Bobbio, Milano, Edizioni di Comunità, pp. 21-39.

CRUZ PARCERO, J.A. (2007), El lenguaje de los derechos. Ensayo para una teoría estructural de los derechos, Trotta, Madrid.

DABIN, J. (2006) [1955], El derecho subjetivo (Trad. de Francisco Javier Osset), Comares, Granada,

DICIOTTI, E. (2004), "Sulla distinzione tra diritti di libertà e diritti sociali: una prospettiva di filosofia analitica", Quaderni costituzionali / a. XXIV, núm. 4, pp. 733-762.

FINNIS, J. (2000) [1980], Ley natural y derechos naturales (Trad. de Cristóbal Orrego), Abeledo Perrot, Buenos Aires.

FERRAJOLI, L. (2011) [2007], Principia iuris. Teoría del derecho y de la democracia (Trad. de Perfecto Ibáñez, Carlos Bayón, Marina Gascón, Luis Prieto Sanchís y Alfonso Ruiz Miguel), Trotta, Madrid.

GROCIO, H. (1987) [1625], Del derecho de presa y Del derecho de la guerra y de la paz. Textos de las obras "De iure Predae" y "De lure Belli ac Pacis" (Trad. de Primitivo Mariño Gómez), Centro de Estudios Constitucionales, Madrid.

GUASTINI, R, (1999) [1996], Distinguiendo. Estudios de teoría y metateoría del derecho (Trad. de Jordi Ferrer), Gedisa, Barcelona.

GUASTINI, R. (2001), "Cinco observaciones sobre validez y derogación", Discusiones, núm. 2, pp. 59-63

GUASTINI, R, (2016) [2014], La sintaxis del derecho (Trad. de Álvaro Núñez Vaquero), Marcial Pons, Madrid.

HART, H.L.A. (1962) [1953], "Definición y teoría en la ciencia jurídica" (Trad. de Genaro Carrió). En: Derecho y moral. Contribuciones a su análisis, Buenos Aires, Depalma, pp. 93-130.

HART, H.L.A. (1982), "Legal Rights". En: Essays on Bentham. Studies in Jurisprudence and Political Theory, New York, Oxford University Press, pp.162-193.

HART, H.L.A. (2009) [1961], El concepto de derecho (Trad. de Genaro Carrió), Buenos Aires, Abeledo Perrot.

HOBBES, T. (1980) [1651], Leviatán, o la materia, forma y poder de una república eclesiástica civil (Trad. de Manuel Sánchez Sarto), Fondo de Cultura Económica, México D.F.

HOHFELD, W. (1992) [1913], Conceptos jurídicos fundamentales (Trad. de Genaro Carrió), Fontamara, México D.F.

IHERING, R. (2011) [1865], La lucha por el derecho (Trad. de Adolfo Posada), Comares, Granada.

KANT, E. (2005) [1797], La metafísica de las costumbres (Trad. de Adela Cortina), Tecnos, Madrid.

KELSEN, H. 1987 [1923], Problemas capitales de la teoría jurídica (Trad. Wenceslao Roces), Porrúa, México D.F. 
KELSEN, H. (1991) [1960], Teoría pura del Derecho (Trad. de Roberto Vernengo), ( $2^{a}$ edición), Porrúa, México D.F..

LAPORTA, F. (1987): "Sobre el concepto de derechos humanos", DOXA: cuadernos de filosofía del derecho, núm. 4, pp. 23-46.

MACCORMICK, N. (1988) [1975], "Los derechos de los niños: una prueba de fuego para las teorías de los derechos" (Trad. Mercedes Carreras y A. Luis Martínez-Pujalte), Anuario de Filosofía del Derecho, núm. 5.

MACKIE, J.L. (2000) [1977], Ética. La invención de lo bueno y lo malo (Trad. de Tomás Fernández Aúz), Gedisa, Barcelona.

NINO, C. (1980), Introducción al análisis del derecho, Astrea, Buenos Aires.

ORUNESU, C. (2012), Positivismo Jurídico y sistemas constitucionales, Marcial Pons, Madrid.

PUFENDORF, S. (2009) [1660], The Books of Elements of Universal Jurisprudence, Liberty Fund, Indianápolis.

RAZ, J. (2001) [1994], La ética en el ámbito público (Trad. de María Luz Melón), Gedisa, Barcelona

RORTY, R. (1991) [1989], Contingencia, ironía y solidaridad (Trad. de Eduardo Alfredo Sinnot), Paidos, Barcelona.

ROSS, A. (1991) [1961], “Sobre los conceptos de 'Estado' y 'Órganos del Estado en Derecho Constitucional". En: El concepto de validez y otros ensayos (Trad. de Eduardo A. Vásquez), México, Fontamara, pp. 73-92.

ROSS, A. (2000) [1969)], Lógica de las normas (Trad. de José S.P. Hierro), Colmares, Granada.

SCHAUER, F. (2004) [1991], Las reglas en juego (Trad. de Claudina Orunesu y Jorge Rodríguez), Marcial Pons, Madrid.

SEARLE, J. (2007) [1969], Actos de habla. Ensayo de Filosofía del Lenguaje, Cátedra, , Madrid.

SEARLE, J. (1997) [1995]: La construcción de la realidad social (Trad. de Luis M Valdés Villanueva), Paidos, Barcelona. (Trad. de Antoni Domènech).

SPINOZA, B. (2003) [1670], Tratado teológico-político (Trad. de Atilano Domínguez), Alianza, Madrid.

STRAWSON, P. (1997) [1992], Análisis y metafísica (Trad. de Nieves Guasch), Paidos, Barcelona.

THOMASIUS, C. (1994) [1705], Fundamentos de derecho natural y de gentes (Trad. de Salvador Rus Rufino y $\mathrm{M}^{\mathrm{a}}$ Asunción Sánchez Manzano), Tecnos, Madrid.

VILLEY, M. (1976) [1968], "El derecho subjetivo en Ihering". En: Estudios en torno a la noción de derechos subjetivos (Trad. de Jorge López), Valparaíso, Ediciones Universitarias de Valparaíso, pp. 223- 238.

VON WRIGHT, G. (1970) [1963], Norma y acción. Una investigación lógica (Trad. de Pedro García Ferrero), Tecnos, Madrid.

WINDSCHEID, B. (1946) [1862], "Lehrbuch des pandektenrechts" (Trad. de Enrique Príncipe y Satorres). En: IHERING, R. La dogmática jurídica, Losada, Buenos Aires. 